\title{
Comparative Study of Left Ventricular Low Wall Motion with Scar Tissue Using 4D Left Ventricular Cardiac Images
}

\author{
Chien-Yi Lee ${ }^{1}$, Yashbir Singh ${ }^{1}$,Michael Friebe ${ }^{2}$, Wei-Chih $\mathbf{H u}^{1}$ \\ ${ }^{1}$ Chung Yuan Christian University, Zhongli District, Taiwan 320 \\ ${ }^{2}$ Otto-von-Guericke-Universität, Magdeburg, Germany \\ weichihhu@gmail.com; weichihhu@cycu.edu.tw
}

\begin{abstract}
Myocardial contraction affects the cardiovascular pumping system, and helps in the early phase to detect abnormalities of wall motion noninvasively. In this research, we designed a program to characterize regional abnormalities because scar tissue is very difficult to identify in normal cardiac CT images. We created 10 frames of a 3D heart model that contains the long axis as reference for predicting the left ventricular wall motion. We tested our 4D cardiac model with scar tissue using non invasive cardiac CT images. Here, four subjects (patients) were involved in this study. Subject 1 and 4 are matching the low motion of surgical area with scar tissue area. Subject 2 found fibrous tissue regions (about 40\%), compared with the 2SD (Standard Deviation) region. The fibrotic area is completely overlapped with a low-motion region which indicates the fibrotic area has a significant correlation with the low wall motion region. This research evaluates low wall motion of the left ventricle and detection of fibrosis regions.
\end{abstract}

Keywords: Left ventricular remodeling; myocardial wall motion; Cardiac imaging; Fibrotic tissue; Noninvasive cardiac evaluation;

\section{Introduction}

Cardiac disease has become a very challenging clinical problem like regional myocardial injury. It is one of the goals of cardiac imaging methods to measure the regional function of the left ventricle (LV). There are many existing techniques to precise and reliable quantitative regional LV function measurements. Most of the standard methods depend on 2D image sequence data [1, 2, 3, 4, 5, 6].Most of the techniques have been used end-diastolic(ED) and end systolic (ES) image frames. While the LV expansion and LV thickening from region to region is indicative of ischemia $[2,4,6]$. Song and Leahy have performed the dense-field optical flow approach to include fluid flow models using 3D datasets [7]. The Goldgof research group followed a shape matching idea which is similar to ours; though they primarily use Gaussian curvature employing conformal stretching models [8]. Pentland and Terzopoulos have been studying non-rigid motion models, using finite element analysis, which might be useful for cardiac analysis. The Ayache group unified these two approaches to segment and track the object simultaneously [9]. A recently proposed technique is the use of phase contrast MR images to decipher local velocity [10]. However, MR images take a long in acquisition and resolution is less than CT images. This can be integrated to estimate trajectories of individual points over time [11, 12]. Our new 
developments could potentially help in curvature estimation, point-wise non-rigid motion tracking, initial quantitative measures of LV motion, thickening, and 3D visualization techniques. The experimental results from real image sequences of multi-phase and multi-slice cardio CT image will be presented, and future research directions will be discussed.

\section{Methods}

This is a Comparative study of low wall motion with scar tissue using 4D left ventricular cardiac images. The methodology has been grouped into various categories:

\section{Data Extraction}

We used Microsoft Windows 7, the 64-bit platform with Intel Celeron CPU G550, memory DDR3 4GB, Visual C ++ 6.0 MFC4.2 for software development and OpenGL library as a tool for the 3D model [13]. Images were obtained from the Philips computerized tomography (CT) instrument provided by the National Institute of Hospital of Yang-Ming University. This study and the informed consent procedure were approved by the Institutional Review Board of National Yang-Ming University Hospital. Each subject has 10 sets of timing frames, including a complete heartbeat cycle. 1 set of 3D cardiac image has 400 slice of cardiac CT images. The scanned image size is $512 \times 512$ (pixels). 1 voxel is $0.165 \mathrm{~mm}^{3}$ $(0.429 * 0.429 * 0.9)$ where $\left(x^{*} y^{*} z\right)$. We used this images to extract edges of endocardium and estimated the endocardium area of LV. In this way, we find the change of edges of wall motion.

The program flow is as follows:

- Read the image into the program, confirm the format for the DICOM (Digital Imaging and Communications in Medicine), the image is $512 \times 512$ gray form stored in the program.

- 3D reorganization and image information is obtained to construct the stereoscopic model of the thoracic area. Set the left ventricular central axis and resample the image [13].

- Perform a manual split using our program at the first slice only then use the regional growth law circle, draw the contour of the left ventricular endocardium.

- The endocardial shape of the LV endocardium is magnified at a variable magnification, and the boundary of the second regional growth is made.

- Repeated manual segmentation is performed to obtain epicardial information. 3D model LV endocardium and epicardium is established by a triangular mesh [14, 15].

Left Ventricular Edge Search

We use manually cutting of LV images from base to apical from the entire data set, selected one image from the dataset and one seeded point to start this process. We obtained the geometrical center that connects each slice on long axis, applied regression calculation to find the line as a reference axis for LV $[16,17]$.

Manually Divide the left Ventricle

In this section, we performed re-sampling of the images to find the short axis, separated LV from aorta

(Fig 1). 
Chien-Yi Lee, Yashbir Singh,Michael Friebe, Wei-Chih Hu; Comparative Study of Left Ventricular Low Wall Motion with Scar Tissue Using 4D Left Ventricular Cardiac Imaaes. Iournal of Biomedical Engineering and Medical Imaging. Volume 5. No 2. Anril (2018), pp 29-39
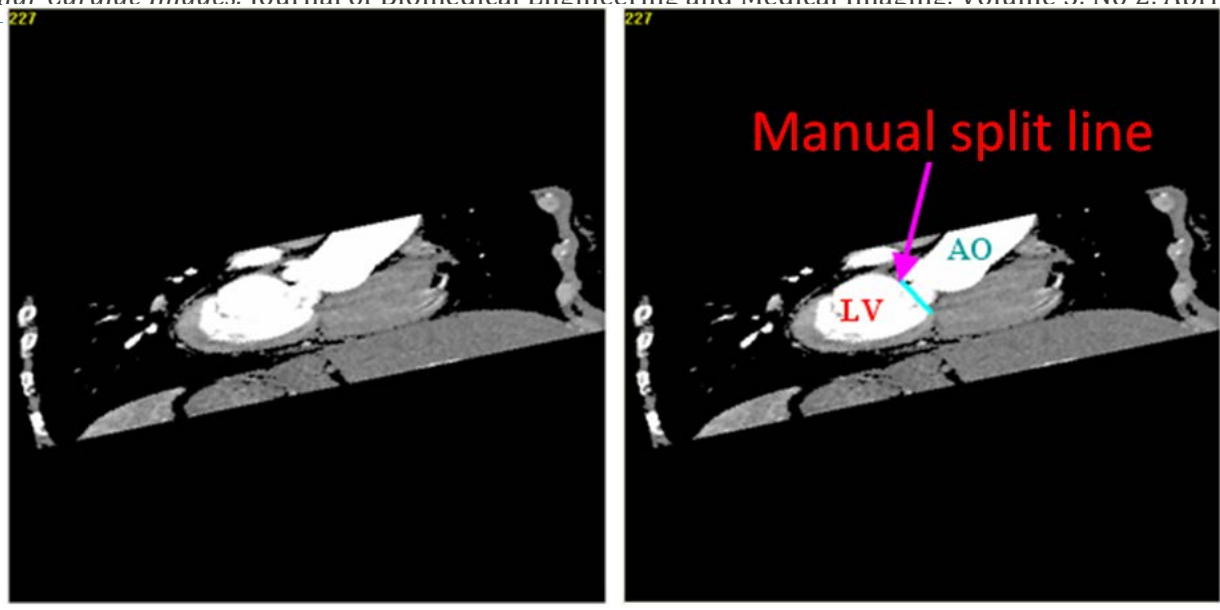

Figure 1.Manually separated line between left ventricle and the aorta.

Regional Growth Circle Selected Endocardium

In this study, the left ventricular endocardial circle is used for the regional growth of the image which is close to the edge of the image. The regional growth of the operation is needed to set up the initial seed point. The initial point of the first slice will be considered as the reference point of the second slice, the second reference point will be set as the seeded region for other layers simultaneously (Fig 2) [16, 17]. It is necessary to confirm that the seeded spots of each layer are in the endometrial region, if not, we need to manually correct the seed points.

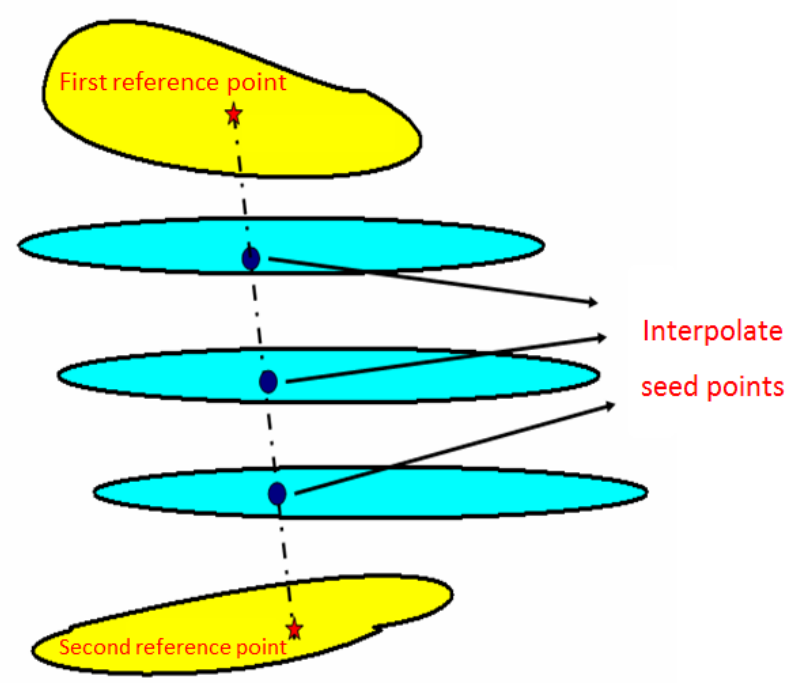

Figure 2.Seeded Point Star from the First Layer and The Last Layer.

\section{Simulation Study}

The simulation model is proposed by Yang Bochuan and Ma Minghui. We obtained LV endocardium and the outer membrane Lattice model at different time of the long axis, calculated the diastolic and systolic period of the radius between the rates of change. After that we obtained the number of myocardial changes at each time. These steps are to obtain the simulated left ventricular at each time. This stereoscopic model is to simulate the changes of the LV in the cardiac cycle and the different values of myocardial changes in each block [18]. 


\section{Triangular Mesh}

We use triangular mesh for LV endometrial and adventitial information 3D. The number of samples for the study of 31 layers of each layer of 30 points a total of 930 points, which defines 1 to 10 layers for the base, 11 to 20 layers is the top layer, the 21-30 layers is the middle layer, and the 31st floor is the reference layer. The sampling method divides the total number of the left ventricles by 31 layers, and stores the coordinate points $(X, Y, Z)$. The simulation is to use these 930 points to do the simulation movement. We calculate the momentum information of the heart when the low amount of movement occurs $[15,19]$.

\section{Simulated Model End of Diastolic Phase}

LV contracts at the centre axis. The endometrial and adventitial information goes the left ventricular central axis to establish a triangular mesh model (Fig 3), which shows the gap between outer membrane model and the endometrial model.10 frame of the triangular mesh is done. In the endometrial triangular mesh model found with the degree of myocardial changes in different levels of red, yellow, green, light blue and dark blue are five colors (Fig.4), and will record the picture in the dark blue block coordinates.

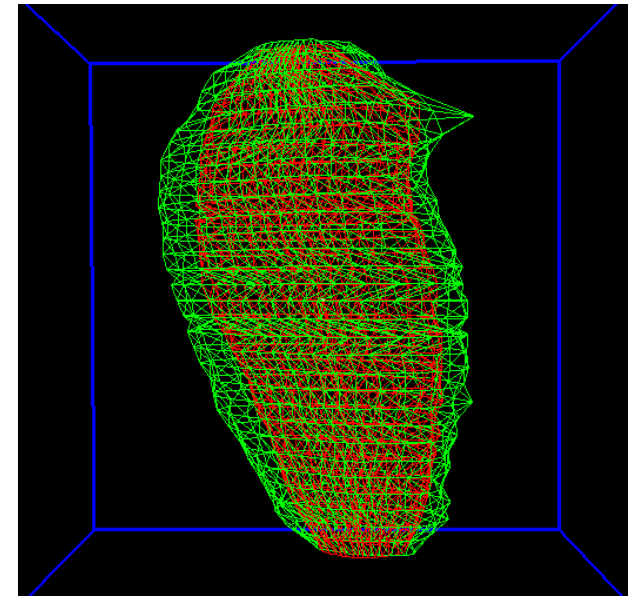

Figure.3 Left Ventricular Triangular Mesh Simulated Model that represents green color for outer membrane model, red for the endometrial model.

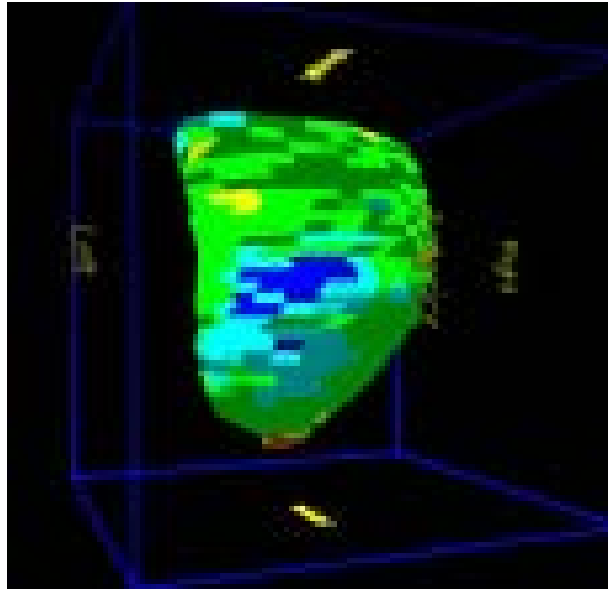

Figure.4 Simulation model of left ventricular motion analysis.

\section{Fibrosis Area Assessment Interface}

This study explores the relationship between regional and motion. Hiroaki and other scholars performed the method using patient's delayed-enhancement images that determined the area of fibrotic tissue. This interface exhibit the calculation within the region of the HU (Hounsfield unit) average or press the single click in the block on the right mouse button, point to the center of the circle to 10 points for the radius of the circle. It automatically calculates the circle within the HU average and pink color display in the circular area (Fig.5). We get the region of interest $\mathrm{HU}$ value which shows region of fibrosis. This experiment performed to find the fibrosis area [20,21]. 


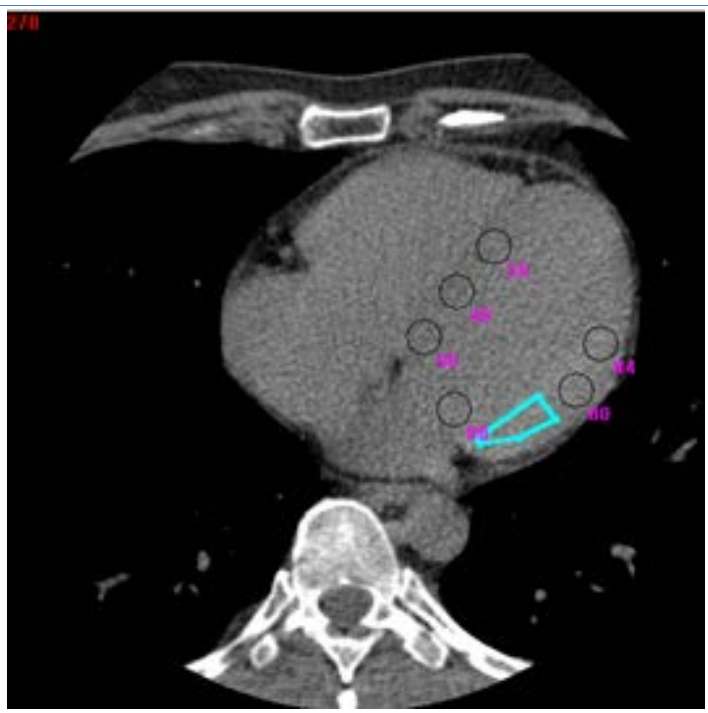

Figure.5 Fibrous area assessment interface (Pink circle area is the HU value of the manual selection area).

\section{Results}

Motion Model Analysis Interface

We determined 10 lattice grid models in the DICOM 0 timing to reconstruct 3D heart volume and executed the program. We get a 4D model of LV motion. This display interface allows the user to observe the three-dimensional model of the inner portion of the left ventricle (Fig 6). The red color model represents a 3D model of the inner membrane, whereas the green color model represents the three-dimensional model of the outer membrane. This simulation system exhibits the LV outer membrane state of motion (Fig.7) which provides better understanding of the movement of the myocardium in the LV. This method is used dynamically to increase the number of left ventricle models in a linear interpolation method.
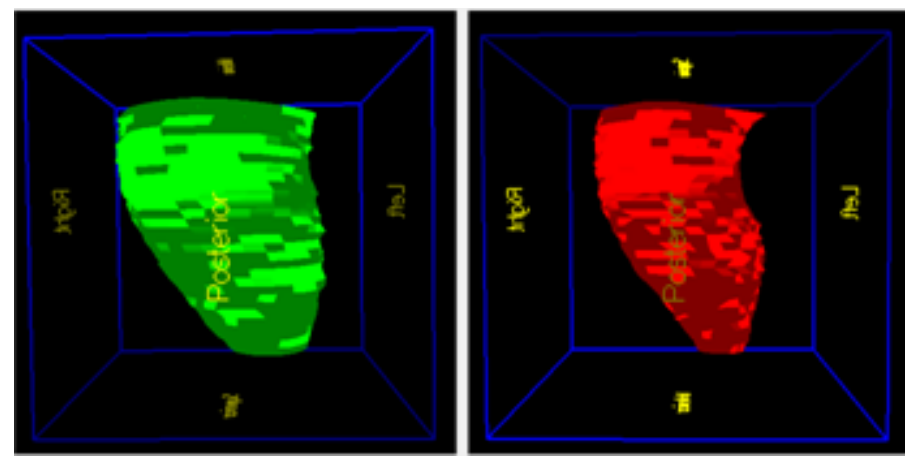

Figure.6 Analog interfaces (The red color model represents inner membrane of 3D, green color model represents outer membrane of 3D). 

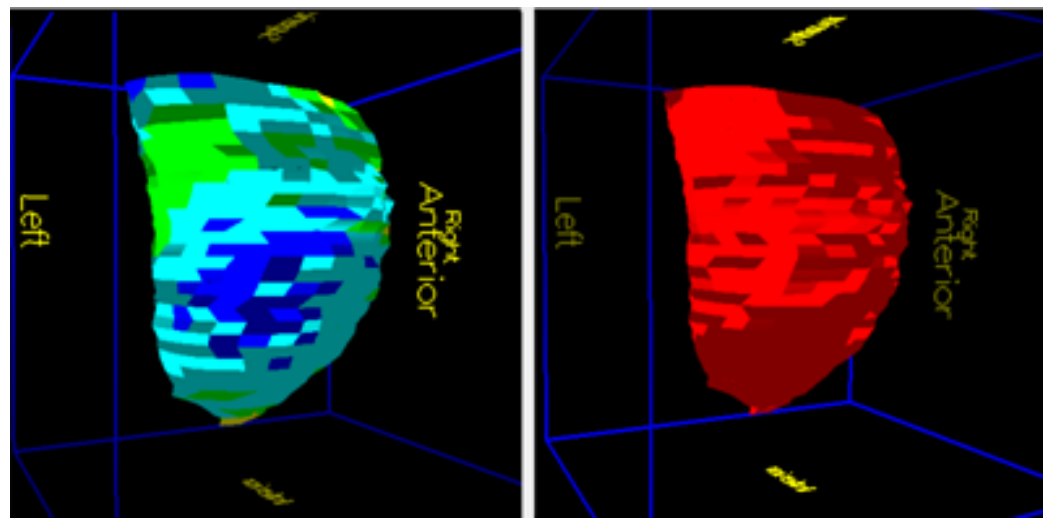

Figure.7 Dynamic interface diagram.

left Ventricular Low Motion Region Assessment

Different color areas represent the difference between the mean left ventricular displacements. The blue is the relative displacement of $2 \mathrm{~mm}$ or less, the light blue is the displacement $2 \sim 3 \mathrm{~mm}$, green is the displacement of $3 \sim 4 \mathrm{~mm}$, yellow is the displacement of $4 \sim 5 \mathrm{~mm}$, red is the displacement of more than $5 \mathrm{~mm}$, and low-motion area is defined as the average amount of difference between the value of less than $2 \mathrm{~mm}$ area. This study re-sampled after the left ventricular model 1 to 10 layers defined as the base layer, 11 to 20 layers is defined as the middle layer, 21 to 30 layers defined as the top layer. The method takes 30 points of sampling points counterclockwise (Fig. 8) (Fig. 9) (Table. 1).

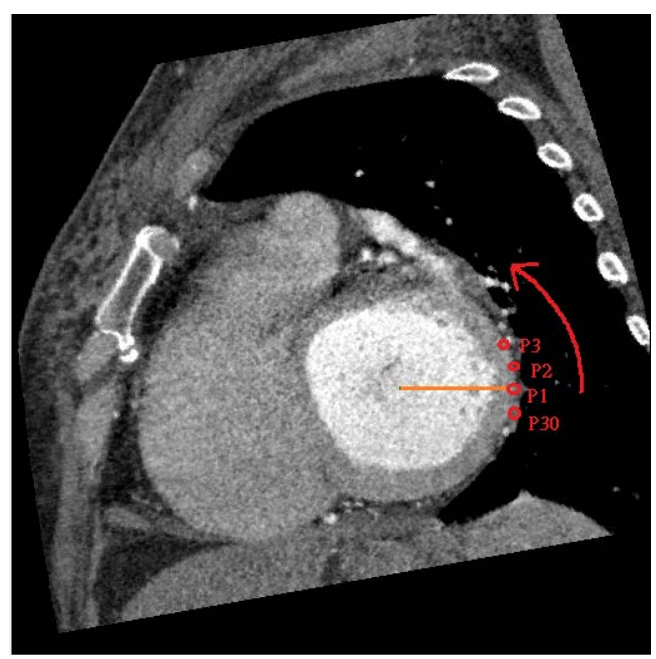

Figure.8 Re-sampling the sampling point.

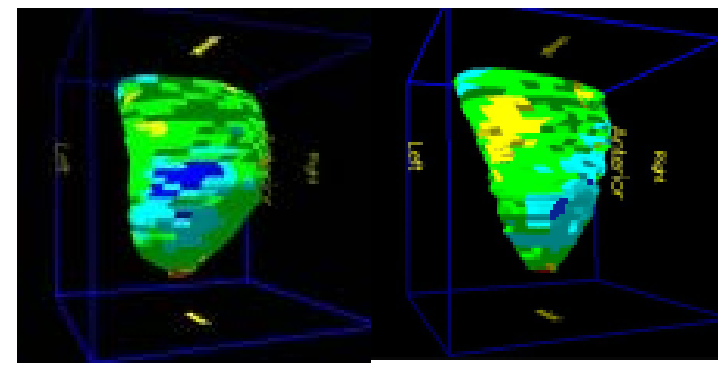

Timing series 0

Timing series 1. 


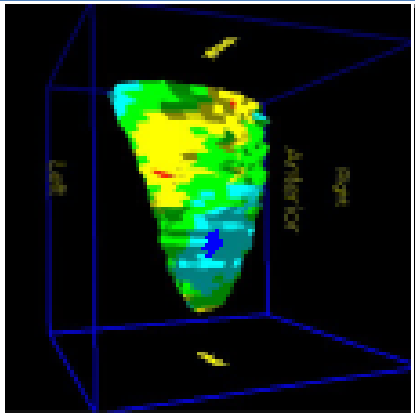

Timing series 2

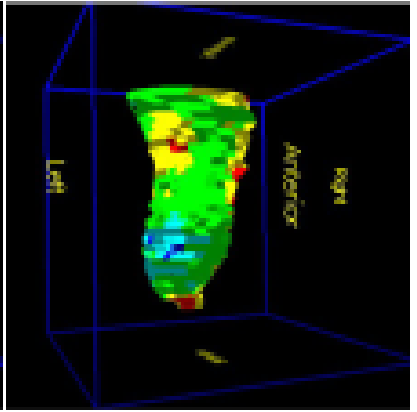

Timing series 3 .

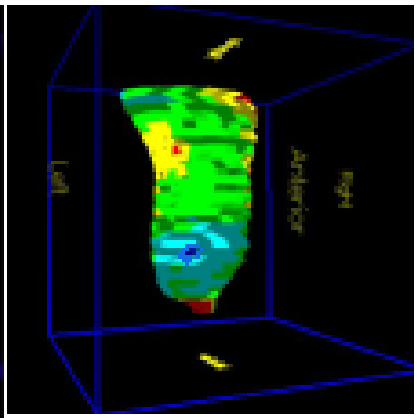

Timing series 5 .

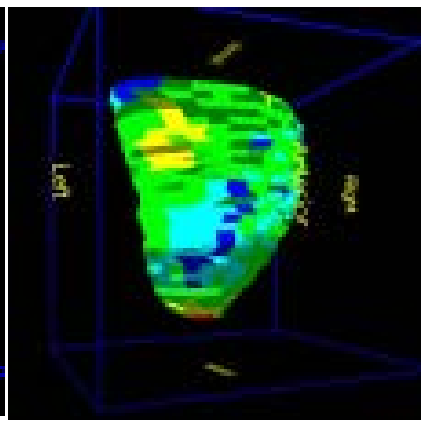

Timing series 7 .

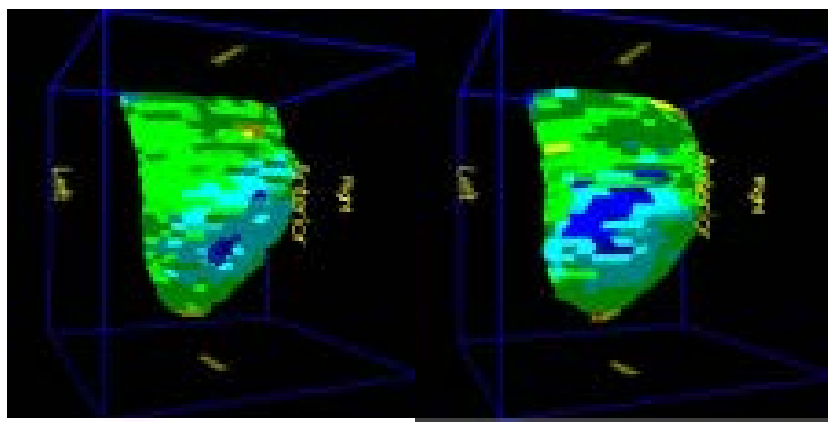

Timing series 8
Timing series 9.

Figure.9 Myocardial changes in the model of subject 1 at every time series. 
Table.1 Analysis result

\begin{tabular}{|c|c|c|c|c|}
\hline Patients & Generation layer & Segment group location & Point shift & Position evaluation \\
\hline Subject1 & $\begin{array}{l}10 \\
11\end{array}$ & $\begin{array}{l}\text { Segment 23, Degree } 276 \\
\text { Segments 24,Degree } 288 \\
\text { Segments 23, Degree } 276 \\
\text { Segments 24, } 288 \text { degree } \\
\text { Segments 25, } 300 \text { degree } \\
\text { Segments 26, } 312 \text { degree } \\
\text { Segments 23, } 276 \text { degree } \\
\text { Segments 24, } 288 \text { degree } \\
\text { Segments 25, } 300 \text { degree } \\
\text { Segments 24, } 288 \text { degree }\end{array}$ & $\begin{array}{l}1.712 \mathrm{~mm} \\
1.784 \mathrm{~mm} \\
1.672 \mathrm{~mm} \\
1.559 \mathrm{~mm} \\
1.588 \mathrm{~mm} \\
1.576 \mathrm{~mm} \\
1.695 \mathrm{~mm} \\
1.701 \mathrm{~mm} \\
1.543 \mathrm{~mm} \\
1.728 \mathrm{~mm}\end{array}$ & $\begin{array}{l}\text { Middle layer } \\
\text { Posterior side }\end{array}$ \\
\hline
\end{tabular}

We observed the low-motion region in the LV (Table .2) and defined 1 to 15 points for the front side (inferior), 16 to 30 points for the back (inferior), each interval is 12 degrees sampling points. In addition, the movement model analysis interface calculated with low sequence (Table.3).

Table. 2 Location of scar tissue in the patients after surgery.

\begin{tabular}{ccc}
\hline Number of patients & Number of layers & area \\
\hline Subject1 & Middle layer & Posterior side \\
Subject2 & Middle layer & Anterior side, posterior side \\
Subject3 & Lower Layer & Posterior side \\
Subject4 & Lower Layer & Anterior side \\
\hline
\end{tabular}

Table.3 Detection of the fibrotic tissue location.

\begin{tabular}{ccccc}
\hline No. of Patients & HU (Avg) & (Std Devi) & Greater than 2SD layers & Area \\
\hline Subject1 & 60.2 & 8.4 & $266 \sim 274$ ( middle layer) & Posterior side \\
Subject2 & 58.7 & 7.9 & $271 \sim 283$ (Middle layer) & Anterior side \\
Subject3 & 71.3 & 12.1 & No & no \\
Subject4 & 64.5 & 9.0 & $302 \sim 309$ (base layer) & Anterior side \\
\hline
\end{tabular}

Determination of left Ventricular Fibrosis Area

We performed patient's surgery of LV site. Using meglumine diatrizoate, we developed enhancement image criteria using Zeinab et al, found the information of the single image with a fibrotic area, scanned the 10-slice and 20-slice myocardial wall area to ensure that the post-operative area in the left ventricle pattern which is defined by the spinal cord for the posterior Side. It contains the lower wall of the hypopharynx, inferior wall, back wall and front of the spinal cord that contains septal, anterior wall and other blocks. We scan whole heart during the systolic phase and find the area which has low changing radius to match the scar area as low wall motion area. The changing radius is $7.76 \%$ which is less than the average changing radius that exhibits low wall motion of LV. The assessment of impaired myocardial function is compared to the central axis angle. Low-motion region is only related to the change in the radius. Therefore, this study shows that the maximum low-motion area, affect the central axis angle changes. The low-volume region is the fibrotic area; results are found (Table. 4). 
Table 4. Descriptive result of Subject 1.

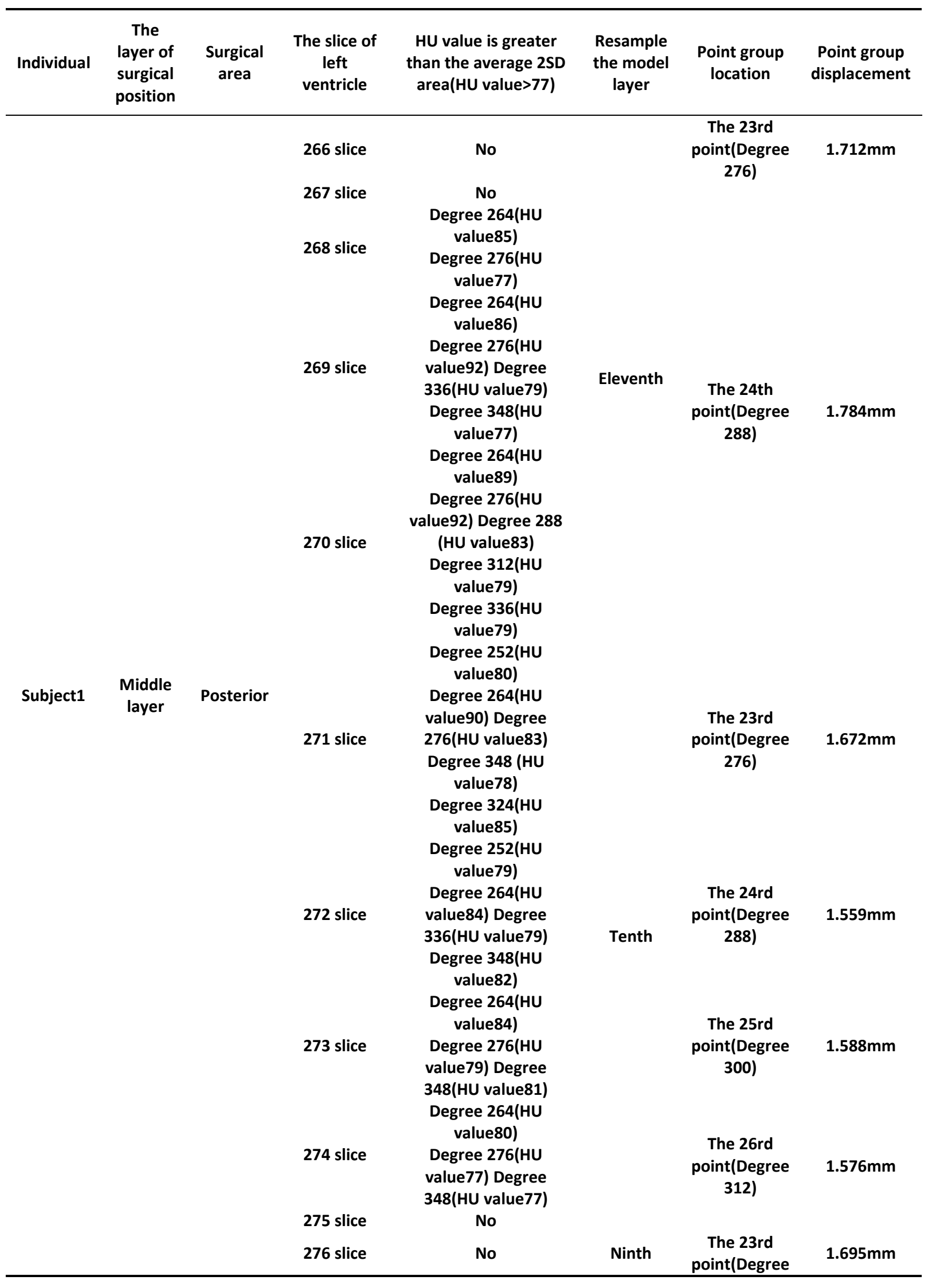




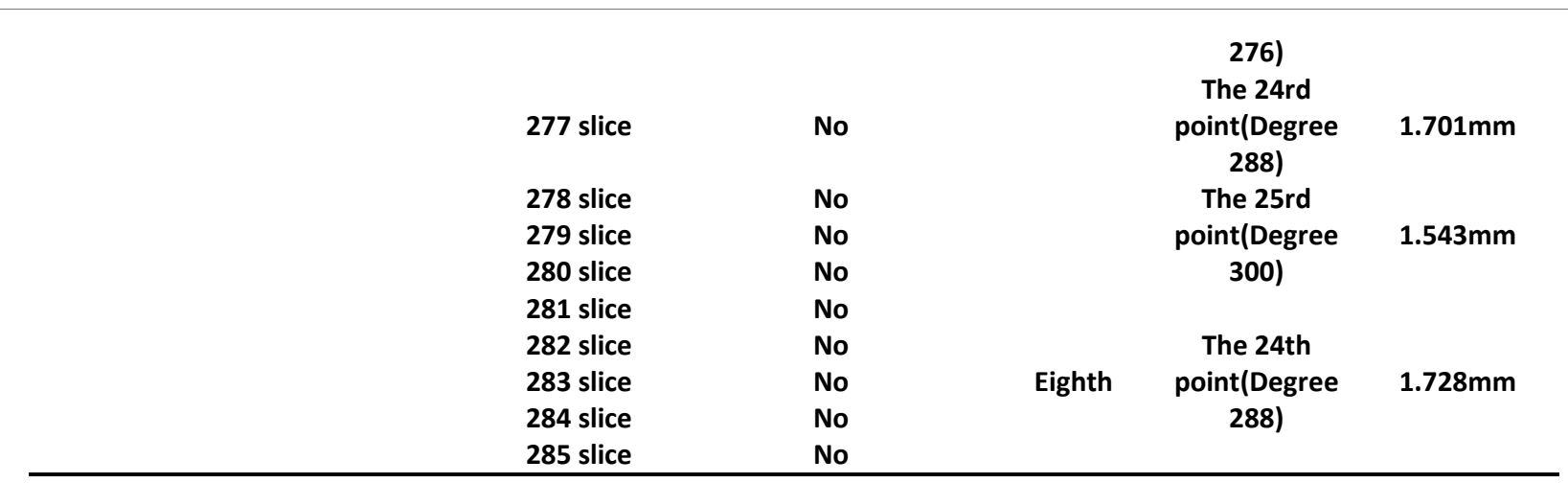

We have four Subjects (Patients), Subject 1and 4 are matching the low motion of surgical area with scar tissue area. Patient 2 has five districts surgical area, the presence of fibrous tissue is only two regions (about 40\%), compared with the 2SD (Standard Deviation) region. The region is higher than the average of 1.6 to 1.8 times the SD value produced. Compared with larger than 2SD region, a slow-motion region found, the fibrotic area completely overlapped with a low-motion region which indicates the fibrotic area. It has a significant correlation with the low-motion region. This study is also found that the fibrosis area is significantly lower than low exercise area. Injured area involved in the movement of adjacent muscles which led to low mobility area than the fibrosis area to the wide. Subject 3 is no fibrotic tissue in this region, as long as the myocardial injury to a certain extent will lead to low motion incidence of the region, but the system can still find the low motion region and position. Only the low-motion area is the medium-risk group in the non-scar area. This study is compared with the fibrotic area method which is proposed by the previous research, our study is to detect the low motion area. It is possible to predict the location of the fibrosis region by irradiating the delayed-enhancement image. The tool can further identify the area of low motion activity without fibrosis, which is performed by the physician for early monitoring, and tracking can provide effective help.

\section{Conclusion}

This research evaluated low wall motion of the left ventricle of the four patients after surgery, myocardial changes analysis and fibrosis of the region confirms the feasibility of the myocardial movement. This method is sensitive to detect the myocardium dysfunction. We can apply this method to find different types of ventricle disease of the cardiomyopathy. This study is included the assessment of myocardial infarction, Cardiac remodeling and assessing the role of fibrotic tissue in the heart. Further research will involve the integration of the heart motion, validating geometrical landmarks and integration of the motion matching.

\section{REFERENCES}

[1] J. Areeda, E. Garcia, K.Vantrain, D. Brown, A. Waxman and D. Berman. A comprehensive method for automatic analysis of rest/exercise ventricular function from radionuclide angiography. Digital Imaging: Clinical Advances in NuclearMedicine, 1982.

[2] E. L. Bolson et al. Left ventricular segmental wall motion - A new method using local direction information. The computer in Cardiology, 1980.

[3] I. Clayton et al. The characteristic sequence for the onset of contraction in the normal left ventricle. Circulation, 1979, 59:671. 
Chien-Yi Lee, Yashbir Singh,Michael Friebe, Wei-Chih Hu; Comparative Study of Left Ventricular Low Wall Motion with Scar Tissue Using 4D Left Ventricular Cardiac Images. Journal of Biomedical Engineering and Medical Imaging, Volume 5, No 2, April (2018), pp 29-39

[4] H. Gelberg et al. Quantitative left ventricular wall motion analysis: A comparison of area, chord, and radical methods. Circulation, 1979,59:991-1000.

[5] C. Slager et al. Quantitative assessment of regional left ventricular motion using endocardial landmarks. JA CC, 1986.

[6] D. Zisserman et al. Cardiac catheterization and angiographic analysis computer applications. Progress in Cardiovascular Diseases, 1983.

[7] S. Song and R. Leahy. Computation of 3D velocity fields from 3D cine CT images. IEEE Transactionson Medical Imaging, 1991.

[8] C. Kambhamettu and D. Goldgof. Point correspondence recovery in non-rigid motion. IEEEComputer Vision and Pattern Recognition,1992, 222- 227.

[9] A. Pentland and B. Horowitz. Recovery of nonrigid motion and structure. IEEE Transactions onPattern Analysis and Machine Intelligence, 1991.

[10] D. Terzopoulos and D. Metaxas. Dynamic 3D models with local and global deformations: deformable superquadrics. IEEE Transactions onPattern Analysis and Machine Intelligence,1991

[11] N. J. Pelc, A. Shimakawaand G. H. Glover. Phase contrast cine MRI. Proceedings of the 8th AnnualSMRM,1989.

[12] J. van Wedeen, G. Holmvang, H. Kantor and T. J. Brady. Measurement of myocardial strain with phase sensitive MR. Proceedings of 2h.e 9th AnnualSMRM,1990.

[13] W. J. Richard S., "OpenGL Super Bible," 1996.

[14] Monga and N. Ayache. From voxel to curvature. IEEE Computer Vision and Pattern Recognition, 1991, 644649.

[15] P. T. Sander and S. W. Zucker. Inferring surface trace and differential structure from 3D images. IEEE Transactions on Pattern Analysis and MachineIntelligence, 1990, 12(9):833-854.

[16] J. Park, D. Metaxas, A. A. Young, and L. Axel, "Deformable models with parameter functions for cardiac motion analysis from tagged MRI data, "IEEE Transactions on Medical Imaging, 1996.

[17] J. Huang, D. Abendschein, V. G. Davila-Roman, and A. A. Amini, "Spatio-temporal tracking of myocardial deformations with a 4-D B-spline model from tagged MRI," IEEE Transactions on Medical Imaging, 1999.

[18] M. F. Smith, "The effect of contraction and twist on myocardial PET and SPECT image resolution: a mathematical phantom study," IEEE transactions on nuclear science, 2000.

[19] L. H. Staib and J. S. Duncan. Deformable Fourier models for surface finding in 3D images. SPIEVol. 1808: Visualization a Biomedical Computing, 1992.

[20] Fearmonti, R., Bond, J., Erdmann, D., \& Levinson, H. A review of scar scales and scar measuring devices. Eplasty, 2010.

[21] Razi, T., Niknami, M., \& Ghazani, F. A. Relationship between Hounsfield unit in CT scan and grayscale in CBCT. Journal of dental research, dental clinics, dental prospects, 2014. 